\title{
Reinventando la práctica docente en la Universidad Estatal a Distancia de Costa Rica: una oportunidad de crecimiento profesional en tiempos de COVID-19
}

\author{
Reinventing teaching practice at Universidad Estatal a Distancia, Costa Rica: \\ an alternative for professional growth in times of COVID-19 \\ Reinventando a prática do ensino na Universidade Estadual à Distância, \\ Costa Rica: Uma opção de crescimento profissional em tempos da COVID-19
}

\author{
Zarelly Sibaja Trejos \\ Universidad Estatal a Distancia. \\ San José, Costa Rica. \\ zsibaja@uned.ac.cr \\ (D) https://orcid.org/0000-0003-1805-3031 \\ Nohemí Hernández Herrera \\ Universidad Estatal a Distancia. \\ San José, Costa Rica. \\ nhernandezh@uned.ac.cr \\ (D) https://orcid.org/0000-0003-3597-9469 \\ Rafael Ángel Granados Portuguez \\ Universidad Estatal a Distancia. \\ San José, Costa Rica. \\ ragranados@uned.ac.cr \\ (D) https://orcid.org/0000-0002-3442-1466
}

Recibido - Received - Recebido: 03 / 08 / 2020 Corregido - Revised - Revisado: 28 / 09 / 2020 Aceptado - Accepted - Aprovado: 02 / 10 / 2020

DOI: https://doi.org/10.22458/ie.v22iEspecial.3245

URL: https://revistas.uned.ac.cr/index.php/innovaciones/article/view/3245

\begin{abstract}
Resumen: Ante la expansión de la pandemia por el virus causante de la enfermedad de la COVID-19 en Costa Rica, el sistema educativo debió enfrentar desafíos en cuanto a la gestión, funcionamiento e implementación de la educación, a nivel nacional. La educación superior no escapó y así debió asumir el reto de proponer estrategias que permitieran la continuación de actividades académicas de naturaleza teórica-práctica dado que lo primordial era y sigue siendo el resguardo de la salud, el bienestar y preservar la vida de las personas estudiantes. La Escuela de Ciencias de la Educación (ECE) de la Universidad Estatal a Distancia se organizó en diferentes comisiones para afrontar tales desafíos. La Comisión conformada por representantes de las carreras de Educación Especial, Preescolar y General Básica, quienes plantean una propuesta para la continuación de las prácticas dirigidas y profesionales de la ECE, la propuesta fue avalada por el Consejo Universitario de dicha universidad y acogida por otras Escuelas para la continuación de los procesos prácticos como opción de graduación según se indica en los distintos planes de estudios. El manuscrito expone los antecedentes que fundamentan la creación de la propuesta académica para la ejecución de las prácticas dirigidas profesionales en la UNED, los principales componentes de esta, la metodología empleada para su diseño y las conclusiones tras este proceso cooperativo entre carreras.
\end{abstract}

Palabras clave: Aporte educacional, necesidades educacionales, innovación educacional, enseñanza y formación, educación a distancia, docencia, práctica pedagógica, COVID-19 


\begin{abstract}
Due to the pandemic caused by the virus that causes the COVID-19 disease, Costa Rica's education system must face challenges regarding education management, operations, and implementation at a national level. Higher Education is not an exception, so taking on the challenge to put forth strategies that allow the academic activities of a theoretical-practical nature to continue considering that what is fundamental is to safeguard the students' lives as well as their health and well-being. The School of Educational Sciences of Universidad Estatal a Distancia is organized in different commissions to face such challenges. The commission made up of representatives of the Special Education, Preschool and Basic General undergraduate programs, laid on the line an approach endorsed by the University Council and welcomed by the other Faculties the proposal for the continuation of the Teaching and Professional Practices of this Faculty, to allow the continuation of the practical processes of the programs as mentioned above that include this process as a graduation option in their study program. The paper sets out the antecedents that support the creation of the academic proposal for the execution of the professional directed practices in the UNED, the main components of this, the methodology used for its design and the conclusions after this cooperative process between careers.
\end{abstract}

Key Words: Educational contribution, educational needs, educational innovation, teaching and training, distance education, teaching, pedagogical practice, COVID-19

Resumo: Diante da expansão da pandemia pelo vírus que causa a doença COVID-19 na Costa Rica, o sistema educacional deve enfrentar desafios em termos de gestão, operação e implementação da educação em nível nacional. O ensino superior não está isento deste desafio e deve, portanto, propor estratégias que permitam a continuação das atividades acadêmicas de natureza teórica e prática, dado que o mais importante é proteger a saúde, o bem-estar e a vida dos estudantes. A Escola de Ciências da Educação da Universidad Estatal aDistancia está organizada em diferentes comissões para enfrentar tais desafios. A Comissão é composta por representantes das carreiras de Educação Especial, Pré-Escolar e Educação Básica Geral, que fazem uma proposta para a continuação do Práticas Docentes e Práticas Profissionais desta Faculdade. A proposta é endossada pelo Conselho Universitário da referida Universidade e é aceita por outras escolas para a continuação dos processos práticos das carreiras que têm este processo como opção de graduação em seu currículo. O manuscrito expõe os antecedentes que sustentam a elaboração da proposta acadêmica para a execução das práticas profissionais dirigidas na UNED, os principais componentes desta, a metodologia utilizada para seu desenho e as conclusões após este processo cooperativo entre carreiras.

Palavras-chave: Contribuição educacional, necessidades educacionais, inovação educacional, ensino e treinamento, educação à distância, ensino, prática pedagógica, COVID-19

\title{
INTRODUCCIÓN
}

La práctica dirigida y práctica profesional en la Escuela de Ciencias de la Educación (ECE) de la Universidad Estatal a Distancia (UNED), constituye la culminación del proceso para obtención del grado académico de diplomado, bachillerato o licenciatura en alguna de las carreras que se ofertan: Administración Educativa, Educación Especial, Educación General Básica I y II Ciclos, Educación Preescolar e Informática Educativa.

Esta práctica dirigida docente o práctica profesional según los distintos planes de estudio y la cantidad de créditos asignados a estas se realiza en contextos educativos reales durante un lapso de 135 a 270 horas distribuidas a lo largo de un semestre, mediante el proceso de acompañamiento técnico entre la persona supervisora y la persona practicante, que aspira a alguno de los títulos académicos antes mencionados. Su objetivo fundamental es evaluar la implementación del ejercicio docente o de la gestión educativa, de las personas estudiantes que han cumplido a cabalidad con la malla curricular propuesta desde cada carrera.

En este proceso, la persona estudiante al concluir su formación profesional como diplomado, bachiller o licenciado en alguno de los campos de la educación que oferta la ECE, tiene el compromiso de devolver a la comunidad docente y estudiantil un servicio profesional de calidad. Actualmente como resultado de necesidad de innovar y preservar la salud, este servicio se brinda mediante la implementación de estrategias mediadas por la virtualidad y educación a distancia. 
Según las estadísticas de matrícula (UNED, 2020) la ECE contó con una población promedio de 606 personas estudiantes que cursaron en los años 2018 y 2019 la práctica dirigida con el fin de culminar su meta de estudio y obtener una certificación que les permitiera entrar al mercado laboral de manera competitiva, con las habilidades y competencias necesarias para el desempeño y atención de calidad a los discentes. En el 2020 la matrícula total en ambos semestres para los niveles de diplomado y bachillerato fue de 560 personas.

Esa posibilidad de conclusión de estudios universitarios a nivel de las carreras de educación se vio amenazada debido a la coyuntura mundial, por la aparición de un virus denominado SARS-CoV-2 que produce la enfermedad respiratoria de la COVID-19, misma que es altamente contagiosa y para la cual aún no hay vacuna preventiva. Esta situación produjo el 11 de marzo la declaratoria de Pandemia Mundial por parte de la Organización Mundial de la Salud (OMS) (Adhanom, 2020), lo que obligó a la mayoría de los países en cuenta Costa Rica a decretar medidas preventivas, entre ellas el aislamiento social. Ello implicó que las personas estudiantes de las prácticas dirigidas y prácticas profesionales no pudieran continuar realizándolas de manera presencial en los contextos educativos.

Ante la situación y en congruencia con el compromiso docente, la ECE se vio en la oportunidad de reinventar procesos educativos que permitieran al estudiantado continuar y concluir con sus estudios universitarios, mediante una opción para realizar las prácticas dirigidas y profesionales manteniendo medidas de distanciamiento social y complementando los procesos con recursos de la educación virtual y a distancia.

Este escrito tiene como objetivo sistematizar la experiencia y ruta seguida para el diseño de la propuesta virtual y a distancia de las prácticas dirigidas y profesionales en la UNED como respuesta ante las exigencias para preservar la salud de su estudiantado y profesorado. Ello permitirá posteriormente evaluar la misma, para enriquecerla o ajustarla según los resultados tras su implementación.

Para el diseño de la presente propuesta fue requerida la constitución de una comisión conformada por representantes de las tres carreras con mayor población estudiantil -estadísticas de matrícula- que cursa las asignaturas de práctica dirigida y profesional, quienes, como parte de los procesos de validación, consultaron en diferentes momentos con las personas encargadas de las demás carreras tanto para la obtención de información preliminar como para la realimentación a la propuesta. También se consultó con docentes supervisores de amplia experiencia en la UNED y que se encuentran laborando en los diferentes contextos educativos, con el fin de valorar la viabilidad y concordancia de las acciones propuestas. Esta consulta se trianguló con la revisión de lineamientos, circulares y comunicados oficiales por parte del Ministerio de Educación Pública de Costa Rica (MEP).

En primera instancia esta comisión realizó un estado del arte en el cual identificó los puntos de convergencia y divergencia entre los distritos planes de estudio, la cantidad de población afectada en el primer semestre la cual ascendía 560 personas. Proyectó según la estadística de años anteriores cual podría ser la cantidad de población estudiantil presumida para el ll semestre 2020 e investigó sobre las posibles opciones de mediación que se ajustaran a la realidad imperante sin poner en riesgo la salud de las personas participantes y garantizando la rigurosidad académica requerida y la prestación de un servicio de calidad.

Fue necesario en el proceso analizar los planes de estudio de cada carrera y las descripciones curriculares de las asignaturas de práctica, indagar sobre los recursos tecnológicos y logísticos con los que contaba en ese momento la universidad y los procesos administrativos y legales requeridos para la aprobación de la propuesta. 
La propuesta final fue también puesta a consideración y realimentación por parte de la Dirección de la Escuela de Ciencias de la Educación que a su vez la compartió con las demás Direcciones de Escuelas y finalmente fue presentada ante el Consejo de Rectoría que tras el análisis de viabilidad correspondiente dio el aval para su implementación.

A continuación, se plantean los acontecimientos que dieron pie a la propuesta que aquí se sistematiza.

\section{DESARROLLO DE LA EXPERIENCIA: PROPOSICIÓN Y ARGUMENTACIÓN}

En marzo de 2020 con la aparición de la primera persona afectada en Costa Rica por el virus de SARSCoV-2 y ante las condiciones impuestas por la urgencia de resguardar la vida, la salud, por medio del distanciamiento social, se hizo necesario de manera intempestiva analizar la situación y establecer rutas de acción emergentes. En ese sentido para Crespo y Garrido (2020) "el factor sorpresa como la desestabilización, el estrés y la reducción de los tiempos que condiciona la toma de decisiones" (p. 13) hace que se instalen nuevas condiciones sociales, en todos los sectores de la sociedad costarricense. Por tanto, la gestión y el funcionamiento de las organizaciones educativas no escapó a esta realidad y debió reorganizarse.

En el marco de lo anterior, la educación costarricense se enfrentó -y continúa haciéndolo- a desafíos en la búsqueda de estrategias para la continuación de la educación costarricense. Todo el sistema educativo debió reinventarse en cuanto a la mediación pedagógica aprovechando tanto los recursos de la educación a distancia como de la educación virtual, para transformar y adaptar las metodologías de trabajo que permitieran afrontar exitosamente esos desafíos.

En cuanto a la educación superior, la UNED no se exime de tales desafíos, y a pesar de que su modelo pedagógico se basa en la educación a distancia, algunos procesos por el carácter de su objeto de estudio se desarrollan preferiblemente de manera presencial en entornos reales, tal es el caso de las modalidades de TFG como prácticas dirigidas y profesionales de esta universidad, mismas que en el Reglamento General Estudiantil se consignan como:

un proceso académico formativo que concluye un plan de estudios para la obtención de un título de grado o posgrado. Este proceso permite a cada estudiante el desarrollo y aplicación de conocimientos, habilidades y destrezas a partir de los conocimientos construidos a lo largo de su formación académica. Propicia la solución de problemas especificos de la realidad desde la disciplina, mediante técnicas y herramientas de investigación. Además, permite comprobar en cada estudiante las capacidades científicas y creativas, en conjunto con sus habilidades para comunicar técnica y asertivamente los resultados de la investigación. (RGE, Art. 81. p. 31)

Para la aplicación de los aprendizajes adquiridos a la largo de la carrera el mismo Reglamento General Estudiantil en sus artículos 85 y 86 incisos a 2 y b4, propone los procesos de práctica dirigida y práctica profesional dirigida, de tal manera que se aclara que estas son actividades académicas en las que de manera innovadora el estudiantado podrá aplicar la teoría procesos metodológicos estudiados a lo largo de la carrera. Estas prácticas se ejecutan individualmente dentro de la dinámica de una organización, empresa o institución ya sean de naturaleza pública o privada, a nivel nacional o internacional, de modo tal que permita evaluar la integración de los aprendizajes con el fin de certificar la competencia profesional futura.

Así las cosas, para el 16 de marzo cuando el gobierno de Costa Rica (Asamblea Legislativa, 2020) decreta Estado de Emergencia Amarilla mediante el Decreto Ejecutivo 42227-MP-S, el estudiantado de las diferentes carreras de educación de la UNED se encontraba laborando en los centros de práctica, realizando 
proceso de diagnóstico institucional, valoraciones iniciales del contexto y la población. Con la declaración de pandemia por parte de la OMS, las autoridades universitarias asumen responsablemente la toma de decisiones en busca del resguardo de la vida, la salud y el bienestar de las personas estudiantes, por lo que se toma el acuerdo en la sesión № 2078-2020 por parte del Consejo de Rectoría de la UNED (2020a), en el que se solicita la suspensión de todas aquellas actividades académicas con carácter presencial, tomando como base los acuerdos de las sesiones 2072, 2073, 2074 y 2075 de año 2020.

La comunidad educativa, responde de manera afirmativa e inmediata al comunicado anterior; suspendiendo temporalmente las prácticas dirigidas y profesionales, lo que generó que las consultas docentes y estudiantado se disparan vía correo electrónico, telefónico y en redes propias de las carreras que tienen en su plan de estudios estas actividades académicas, ante lo que se plantea a la dirección de la ECE la posibilidad de diseñar una propuesta alternativa.

En ese momento según las circunstancias no era posible una solución inmediata ante la congelación de las actividades académicas presenciales, pero el ingenio se hace presente en las personas que conforman el equipo de la ECE, y desde la Dirección de dicha Escuela se lanza la estrategia de la creación de comisiones específicas para hacerle frente a la nueva situación y de la que se derivan acciones específicas para la creación de nuevos escenarios educativos. Esa medida toma en cuenta a todas las personas que trabajan en esa dependencia con el fin de favorecer el aprovechamiento al máximo de los recursos. De esa forma, el 31 de marzo nace la Comisión de Prácticas enfocada a la elaboración de un protocolo de contingencia que permitiera al estudiantado poder continuar de manera segura y con rigurosidad académica su proceso de práctica, esta propuesta debía incluir también de manera preventiva opciones para quienes iniciarían la práctica en el Il semestre de 2020.

La Comisión de Prácticas revisó el diseño curricular de las carreras de la Escuela Ciencias de la Educación que tienen prácticas dirigidas y prácticas profesionales en su plan de estudio, las cuales son Informática Educativa, Educación General Básica I y II Ciclos, Educación Preescolar, Administración Educativa y Educación Especial y determinó aspectos en los coincidían. En la tabla 1 se sintetizan los mismos.

TABLA 1

Acciones relacionadas con los procesos de práctica

\begin{tabular}{|c|c|c|c|c|c|}
\hline Carrera & Orientación & Modalidad & Horas & Créditos & $\begin{array}{l}\text { Organización } \\
\text { del proceso }\end{array}$ \\
\hline Administración Educativa & Práctica & Híbrida & 135 & 3 & En etapas \\
\hline Educación Preescolar & Práctica & Presencial & 180 & 4 & En etapas \\
\hline Educación Especial & Práctica & Presencial & 270 & 6 & En etapas \\
\hline $\begin{array}{l}\text { Educación General Básica I y II Ciclos } \\
\text { (Práctica I) }\end{array}$ & Teórico-Práctica & Híbrida & 142 & 6 & En etapas \\
\hline $\begin{array}{l}\text { Educación General Básica I y II Ciclos } \\
\text { (Práctica II) }\end{array}$ & Teórico-Práctica & Híbrida & 270 & 6 & En etapas \\
\hline Informática Educativa & Práctica & Híbrida & 135 & 3 & En etapas \\
\hline
\end{tabular}

Fuente: Elaboración propia (2020).

Según la información anterior, las carreras de la ECE subdividen la práctica en diferentes etapas, solo para efectos de la organización y desarrollo del proceso. En términos generales, una vez concluidos los procesos administrativos de prematricula y matrícula, todas aplican etapas como:

- Inducción a las personas estudiantes que cumplen con los requisitos

- Observación

- Diagnóstico o valoración 
- Planificación de propuestas educativas

- Ejecución de las propuestas

- Sistematización del proceso de práctica

Además, todas las carreras desde la Educación a Distancia tienen como fundamento que la construcción del aprendizaje es una responsabilidad personal del estudiantado y el rol que ejerce la persona docente es de mediadora, facilitadora y acompañante. De esta manera, al realizar un trabajo de campo bajo supervisión, la persona estudiante se compromete a acatar los lineamientos propuestos tanto en el Reglamento General Estudiantil como los que emanen de las autoridades universitarias y todos aquellos pertenecientes al Ministerio de Educación Pública (MEP) o centro educativo donde se realice el trabajo de campo por medios remotos, ya sea de manera sincrónica o asincrónica.

Las herramientas que se utilizarían debían permitir la interactividad propia de un proceso de práctica dirigida o profesional, pero ahora, permeada de la progresiva virtualización de los procesos de enseñanza y aprendizaje. En este sentido vale la pena referir a lo que Ruiz-Corbella y García-Gutiérrez (2020), han denominado Aprendizaje-Servicio Virtual (ApSv), mismo que,

no supone una mera digitalización de procesos que también se podían realizar de manera presencial y que ahora, gracias a las tecnologías, se llevan a cabo virtualmente (...) no como trasposición del presencial, sino reflexionar expresamente como sería su desarrollo en un ambiente virtual, en el que se plasma la singular y permanente continuidad online - offline. (Ruiz-Corbella y García-Gutiérrez, 2020, p. 187).

Por consiguiente, fue necesario reflexionar y conocer a fondo la información pertinente, las condiciones y los objetivos que se deseaban alcanzar en materia de formación curricular apegado al contexto de cada población beneficiaria y carrera que se cursa, con el fin de identificar las estrategias de virtualización en conjugación con las ya empleadas técnicas de educación a distancia que se debía incluir en la propuesta.

Aunado a los aspectos curriculares propios de cada carrera, el aprendizaje y uso de herramientas tecnológicas se convirtieron en competencias necesarias para lograr y alcanzar metas de y con las personas estudiantes de las prácticas. La propuesta para la continuación de la práctica profesional y práctica dirigida, entonces, debía conjugar todas las habilidades y conocimientos que las personas estudiantes habían desarrollado a lo largo del curso de las asignaturas que cubren la malla curricular y, en cierta forma, la implementación del modelo pedagógico que la misma universidad ha empleado en su formación docente.

Para el desarrollo de la educación a distancia unimodal o tradicional, según apunta García (2018), las estrategias de mediación están basadas en soportes como el texto impreso, el uso del teléfono o correo postal y los programas de radio, en los que el diálogo mediado entre docentes y estudiantes es el principal elemento de interrelación. Sin embargo, esto ha venido cambiando y cada vez son más los docentes, instituciones educativas públicas y privadas que apuestan por la enseñanza flexible y abierta que la educación a distancia permite. Asimismo, agradecen la posibilidad de disponer de escenarios educativos que contemplen el uso de herramientas virtuales de inmersión diseñadas para la gestión y desarrollo de cursos y programas.

En el encuadre anterior, la propuesta debía presentar ideas generales para que cada carrera pudiera ajustar según la naturaleza teórico-práctica de la misma, incluso permitiera plantear varias opciones que se ajustasen a las realidades de la población estudiantil y las zonas donde estas personas se encontraban dadas las características de conectividad existentes. 
En adendum a lo anterior y tomando en cuenta todos los recursos institucionales como: acceso a la plataforma educativa de Moodle, que en la universidad recibe los nombres de EducaU, AprendeU y EstudiaU, centros universitarios con laboratorios de equipo informático, el programa de préstamo de tabletas para el proceso educativo, el uso licenciado de Office $365^{\oplus}$ para el estudiantado, los recursos audiovisuales disponibles, las herramientas de video comunicación en uso como Microsoft Teams ${ }^{\circledast}$, BigBlueBottom, Scopia ${ }^{\oplus}$, Zoom ${ }^{\circledast}$, entre otros muchos aspectos. Se planteó ante las autoridades institucionales un plan innovador y con suficiente amplitud para el desarrollo de las prácticas dirigidas y profesionales en la Universidad Estatal a Distancia.

El 25 de mayo del 2020 en sesión de Consejo de Rectoría (2020b) 2086-2020, Artículo l, inciso 17), se acordó: "Acoger la propuesta presentada para la realización de Prácticas Supervisadas o Docentes, elaborada por la Escuela Ciencias de la Educación (REF. 1298-20)" (Consejo de Rectoría, 2020). A continuación, se procede a resumir la propuesta de plan piloto presentada en su momento por Sibaja, Hernández y Granados (2020), misma en la que se plantearon varias etapas para la ejecución de la práctica mediante tres posibles escenarios, se enlistaron los recursos que serían requeridos, la ruta que se debía seguir antes, durante y después de la implementación, así como las acciones que convenía tomar en cuenta con cada persona involucrada en el proceso.

El plan piloto aprobado propuso modalidades o escenarios educativos en los que se podrían desarrollar los ajustes a las prácticas dirigidas y profesionales en curso y las que se debían iniciar para el II semestre 2020.

Escenario 1: Modalidad distanciamiento social. Implicaba la realización del proceso de práctica por medio de la educación a distancia y presencial aplicando las medidas generales del distanciamiento social. Esta en concordancia con las acciones tomadas por el Ministerio de Educación Pública que planteó un acompañamiento al estudiantado por medio del desarrollo de Guías Autónomas de Trabajo, elaboradas por el cuerpo docente y complementadas con apoyos virtuales en las situaciones que estos fueran posibles.

Para la Dirección de Educación Técnica y Capacidades Empresariales (DETCE) del Ministerio de Educación Pública (2020), una guía de trabajo autónomo es una herramienta pedagógica bajo la filosofía de aprender a aprender; que busca desarrollar competencias de autoaprendizaje gracias al diseño e implementación de actividades y metodologías que bajo criterios de desempeño previamente definidos potencian el logro de los resultados de aprendizaje establecidos en el programa de estudio.

Escenario 2: Modalidad simulada exclusivamente. Propuso la realización del proceso de práctica por medio de contextos, escenarios y acciones educativas simuladas exclusivamente. En los cuales se daría el modelaje de situaciones educativas por parte del equipo de profesores y profesoras supervisores, con el fin de que el estudiantado pudiera realizar análisis y resolución de situaciones de vida (estudios de casos, resolución de problemas). Algunas acciones que permiten el desarrollo de estrategias para esta modalidad pueden ser:

- Creación de diferentes actividades de simulación en las que se deberá ir aumentando paulatinamente el nivel de complejidad.

- Descripción de cada situación y las acciones que se esperaría realice en cada una de ellas, junto con las rúbricas de calificación correspondientes.

- Para el análisis de situaciones de vida (estudios de casos, resolución de problemas) que se planteen; cada estudiante deberá revisar detenidamente la situación que se le presente y propondrá las recomendaciones de solución pertinentes según lo estudiado a lo largo de la carrera, ello puede incluir elaborar informes de valoración inicial o final y formular propuestas de abordaje educativo y/o profesional o de implementación en un centro educativo o comunidad según se requiera. 
Escenario 3: Modalidad híbrida. Presentaba la posibilidad de realizar el proceso de práctica por medio de contextos, escenarios y acciones educativas simuladas, más la puesta en pericia de las propuestas educativas o profesionales exclusivamente en etapas de ejecución o aplicación. Estas acciones de aplicación podrían realizarse mediante entornos virtuales o en contextos de presencialidad en caso de que las medias sanitaras así lo permitieran.

Los escenarios planteados se realizarían con las acciones correspondientes que garantizarán un buen desarrollo del proceso y el resguardo del bienestar y seguridad del estudiantado.

Como complemento al diseño de los tres posibles escenarios fue necesario apuntar los recursos que serían necesarios para la implementación, entre ellos:

- Dotación de correo electrónico institucional para el profesorado de práctica de las diferentes ofertas académicas. Esto por cuanto el personal en su mayoría es nombrado por servicios profesionales y no cuentan con correo de cuenta institucional.

- Apertura de entornos educativos en EducaU para todas las asignaturas de práctica de la Escuela de Ciencias de la Educación, ya que al momento de la pandemia y al ser la práctica presencial, no todas contaban con dicho recurso.

- Uso compartido en el mismo dominio del Office $365^{\circledR}$ para personal docente y estudiantado de manera que tuvieran acceso fácil a la herramienta de Microsoft Teams ${ }^{\circledR}$, o en su defecto que se tuviera acceso a licencias de otros recursos de videoconferencia como Zoom ${ }^{\circledast}$.

- Realizar ajustes mediante adendum a las Unidades Didácticas Modulares o Manuales de los procesos de práctica de las diferentes carreras de la ECE.

- Que tanto el estudiantado como el cuerpo docente tuviera acceso o conexión a internet. Para ello la universidad cuenta con laboratorios de cómputo en todas sus sedes y se aprobó además un subsidio para el pago de internet al estudiantado en situación de pobreza y vulnerabilidad.

- Acceso a computadora, tableta o teléfono inteligente con capacidad de audio y video, en los que se pudiera correr el Office $365^{\circledR}$ que ofrece la UNED a su estudiantado y profesorado.

Ya teniéndose planteada la oferta de modalidades o escenarios e identificados los recursos básicos para su ejecución, se debía establecer la ruta a seguir en lo que respecta a las acciones previas por parte de las carreras:

- Realizar el comunicado oficial en la página WEB y por los medios electrónicos disponibles, de la modalidad de práctica que se ofertaría para el II semestre de 2020. Esto con el fin de anticipar al estudiantado y mantener congruencia con el supracitado artículo 82 del Reglamento General Estudiantil.

- Publicitar las fechas de matrícula de las prácticas en las redes sociales de las mismas y de la ECE para captar la mayor cantidad de estudiantes que cumplen con los requisitos.

- Establecer convenios con centros educativos, organizaciones u otros en los que el estudiantado pueda realizar la Práctica. Para mitigar posibles afectaciones, se plantean algunas alternativas dirigidas a facilitar la disponibilidad de instituciones educativas aliadas que pudieran constituirse en centros de práctica, entre las opciones que se plantearon en la propuesta se enlistan:

a) Establecer convenios con programas educativos privados que estén dispuestos a aportar sus recursos tecnológicos y población estudiantil a cambio del apoyo que implica contar con estudiantes practicantes y su correspondiente persona supervisora. 
b) Establecer convenios con instituciones públicas que requieran de apoyo de estudiantes practicantes UNED y que cuenten con los recursos y organización técnica requerida para este proceso.

c) Ubicar estudiantes practicantes con grupo de estudiantes de la persona supervisora de práctica.

d) Trabajar con un grupo de estudiantes que se haya inscrito voluntariamente para formar parte de las personas beneficiarias de los servicios remotos de la ECE. Para lo cual la ECE en la figura de la carrera deberá hacer una convocatoria que incluya los requisitos y beneficios con los que contarán las personas voluntarias.

e) Coordinación con docentes egresados de la UNED para que apoyen autorizando la realización de prácticas dentro de sus grupos de trabajo.

Una vez cubierta la etapa de divulgación y preparación de ambiente, se debían realizar acciones propiamente administrativas durante el proceso de prematricula que permitieran conocer la realidad de las personas que matricularían y prever los ajustes a requerir.

En el proceso de prematrícula

Con las personas estudiantes:

1. En concordancia con el artículo 82 del Reglamento General Estudiantil, se debía comunicar a las personas estudiantes de las carreras de la ECE acerca de las adaptaciones relacionadas con el proceso de práctica para el segundo semestre 2020.

2. Por medio del correo electrónico se consultaría al estudiantado para determina el nivel de accesibilidad a herramientas informáticas y a internet del que disponían.

3. Valoración por los departamentos competentes (ofician de becas y bienestar estudiantil), la opción de beca según condición socioeconómica, para contar con apoyo de internet por seis meses para las personas que iniciarán la práctica en el Il semestre.

4. Autorización de la matrícula a las personas estudiantes que ante la situación atípica paliativa contaran con los requisitos académicos y los recursos tecnológicos requeridos.

Con las personas docentes:

1. Se requería también realizar un diagnóstico de las competencias tecnológicas de las personas supervisoras de práctica. Además de determinar la disponibilidad de estas para participar del plan piloto e identificar los requerimientos de capacitación para ejecutar la propuesta.

2. Sería necesario determinar el equipo académico y directores o docentes supervisores que se iba a requerir para la realización de las acciones de entornos simulado o aplicación en situaciones reales posibles según los lineamientos de salud vigente.

3. Era también pertinente la identificación, creación y facilitación material autodidacta sobre el uso de herramientas de comunicación para complementar el desarrollo de competencias al respecto.

Con los recursos institucionales:

1. Era necesario solicitar entorno educativo en EducaU (en el caso de que aún no se contara con el mismo) y uso compartido del Office $365^{\circledR}$ tanto de tutores como de estudiantes, de manera que ambas poblaciones hicieran acopio del mismo dominio, eso facilitaría el trabajo cooperativo. Para ello era fundamental que al personal que se le contratara por servicios profesionales también tuviese acceso a las mismas herramientas. 
Para dar inicio al curso lectivo, una vez terminado el período de matrícula semestral, se debía trabajar dos procesos de inducción, con el fin de generar un ambiente propicio y favorecer la coordinación docente-estudiantado, a saber:

Inducción al estudiantado:

- Diseño y comunicado de lineamientos generales sobre la práctica.

- Capacitación o familiarización con los recursos digitales que se emplearían en el proceso de práctica.

- Comunicado del nombre y contacto de la persona docente o directora supervisora asignada.

Inducción al profesorado:

- Creación de usuarios de correo electrónico y establecimiento de las herramientas de video comunicación o plataforma que se utilizaría para la mediación de la práctica.

- Capacitación o familiarización con los recursos digitales que se emplearían en el proceso de práctica.

- Comunicado de los nombres y datos generales del estudiantado asignado.

Por otra parte, se plantearon acciones generales relacionadas con los procesos de práctica de las carreras de la ECE en sus diferentes etapas. En el mismo se tomaron en cuenta los escenarios descritos anteriormente, los cuales no son excluyentes entre sí, al contrario, estos se podrían complementar de forma tal que en la experiencia educativa se vea favorecido el aprendizaje.

Los nombres con los que se denominaron las etapas de la práctica en los cuadros dos y tres, fueron generales para que pudiesen ser aplicables a los procesos particulares de cada carrera tanto de la ECE como de otras dependencias de la UNED.

De este modo, indiferentemente del escenario seleccionado, se plantearon cinco etapas básicas: 1. Observación, 2. Valoración diagnóstica y fundamentación teórica, 3. Planeamiento u organización de propuestas pedagógicas, 4. Ejecución o aplicación de la propuesta, 5. Sistematización y evaluación.

Para todas ellas se propusieron algunas alternativas o sugerencias en cada uno de los tres escenarios planteados, a saber: distanciamiento social, exclusivamente simulado o modalidad híbrida en la que se podía conjugar acciones simuladas y aplicación directa en entornos reales mediante estrategias de virtualidad y distanciamiento social. En las tablas 2 y 3 se detallan esas acciones que eran posibles, sin que ello limitara que las carreras según su objeto de estudio pudieran plantearse otras opciones. 
TABLA 2

Acciones relacionadas con los procesos de práctica según el escenario número dos sobre situaciones de simulación

$\begin{array}{cc}\text { Etapa } & \begin{array}{c}\text { Etapa de valoración } \\ \text { diagnóstica y } \\ \text { de observación } \\ \text { fundamentación teórica }\end{array}\end{array}$

1. Realizar análisis de situaciones de vida (estudios de casos) y propuestas de resolución de problemas como ejercicio académico. Estas pueden ser por medio de videos, narraciones de audio o documentos escritos. lizada sobre las temáticas que competen al área disciplinar con el fin de elaborar un marco teórico que sustente la propuesta de práctica y le permita resolver las diferentes situaciones que se le plantearan a lo largo del semestre.

2. Revisión de recursos educativos (videos, multimedia, podcast y otros) para fortalecer el análisis de estrategias y el desarrollo te-
1. Revisión de bibliografía actuamático de la disciplina.

3. Elaboración de instrumentos de valoración con su correspondiente fundamentación a modo de marco metodológico del proceso diagnóstico ya sea por medio de encuestas, entrevistas en línea, sesiones de trabajo en línea, exploración documental y otros. Todo ello para la elaboración de:

a. Diagnóstico institucional comunitario, interno administrativo, de recurso humanos, monografía del centro educativo.

b. Valoraciones integrales del estudiantado.

c. Valoraciones de materiales educativos existentes

d. Valoraciones de programas de las asignaturas básicas y sus particularidades.

e. Valoración de temas de capacitación que requiere una población particular.

4. Elaboración de informes de valoración o resultados de los diagnósticos e indagaciones realizadas.

5. Indagar sobre las reseñas históricas de las comunidades en las que están inmersos los centros de práctica.

6. Investigar información del centro de práctica por medio de las monografías.

\section{Planeamiento u organización de propuestas pedagógicas}

Primer escenario:

Resolución de situaciones de simulación

a. Trabajar análisis de situaciones de vida (casos) que vayan aumentando en nivel de complejidad, los cuales deben ser resueltos de forma objetiva. Cada estudiante practicando, debe revisar detenidamente la situación que se le presenta y proponer las recomendaciones de solución pertinente según lo estudiado a lo largo de la carrera, ello puede incluir elaborar informes de valoración inicial o final y formular propuesta de abordaje educativo.

b. Simulaciones de situaciones o funciones administrativas que se pueden dar en centro educativos con Juntas Administrativas, comunidad, familia, docentes, funcionarios administrativos, estudiantes, autoridades ministeriales u otros gestores educativos.

c. Simulaciones de clases informáticas o aplicaciones tecnológicas que pueden desarrollarse en el centro educativo.

\section{Ejecución o aplicación de la propuesta}

Primer escenario:

Resolución de situaciones de simulación

1. Desarrollar sesiones de simulación de problemas concretos de manera que se logren verificar aspectos relacionados con el perfil de salida de cada carrera.

2. Elaboración de recursos pedagógicos, para el abordaje de contenidos, en apoyo a padres y madres de familia de niños y niñas que estudian desde casa.

a. Hacer videos para las conmemoraciones escolares

b. Hacer materiales interactivos o videos que se puedan presentar offline y online (audios, videos, material interactivo) en formatos distintos siguiendo los principios del DUA

c. Construcción de matrices, bases de datos, infografías como apoyo a la administración

d. Diseño de talleres de capacitación virtual, dirigido a padres y madres de familia, docentes $y$ administrativos.

e. Creación de programas radiofónicos para capacitación a padres y madres de familia, docentes y administrativos.

f. Creación de programas radiofónicos para el abordaje de contenidos con niños y niñas que estudian desde casa.

\section{Sistematización y evaluación}

1. Recopilación de evidencias del proceso de práctica.

2. Elaboración de informe final de práctica (TFG)

3. Presentación del informe final de práctica (TFG) 
TABLA 3

Acciones relacionadas con los procesos de práctica según los escenarios que impliquen aplicación y distanciamiento social

\section{Etapa de observación \\ Etapa de valoración diagnóstica y fundamentación teórica \\ Planeamiento u organización de propuestas pedagógicas}

1. Debido a que en esta modalidad la observación no se plantea realizar in situ se pueden utilizar otras herramientas como las citadas en el cuadro dos: videos, narraciones de audio o documentos escritos.
1. Elaborar y aplicar entrevista (vía telefónica o virtual) con el fin de recolectar información del centro educativo o del estudiantado

2. Observar videos, materiales o informes que docentes titulares o docenes supervisores y supervisoras, han grabado de sus clases, previa coordinación, para el análisis de estos.

3. Investigar los contenidos que se van a desarrollar para la etapa de ejecución con la persona titular del centro de práctica

4. Elaborar el planeamiento o propuesta pedagógica con sus correspondientes materiales para ejecutar con las personas con las que se realiza la práctica

5. Determinar el procedimiento horario y herramienta informática por medio de la cual se va a ejecutar el planeamiento o la propuesta pedagógica

6. Establecer la forma en la que se va a recolectar la evidencia de que se está ejecutando el planeamiento o la propuesta pedagógica.
1. Planificar el desarrollo de contenidos temáticos, utilizando guías de trabajo para los encargados y /o padres de familia del estudiantado con el que se realiza la práctica.

2. Determinar en coordinación con las personas supervisoras y titulares del centro de práctica, la metodología, para el envío de los materiales a la población con la que se realiza la práctica.

3. Establecer las herramientas de videocomunicación para el desarrollo de la propuesta pedagógica $y$ planeamientos. Para ello se debe contar con el debido consentimiento informado de la familia o encargados legales del estudiantado y con los recursos virtuales requeridos.

4. Elaboración de materiales para la ejecución de las guías de trabajo.

\section{Ejecución o aplicación} de la propuesta

Para la ejecución de propuestas pedagógicas que requieran uso de guías de trabajo y su respectiva ejecución con los padres de familia:

- Se establece un horario en el que se va a trabajar la explicación de contenidos (por la herramienta de video comunicación acordada según se indica en el punto 3 de la columna de planeamiento $u$ organización de propuestas pedagógicas).

- Se envía con anticipación el planeamiento o guías de trabajo (lo que se va a trabajar)

Se ejecuta la explicación temática de lo que se va a desarrollar y se establecen las estrategias para reco ger la evidencia de que se está trabajando en ello para la evaluación.

En esta modalidad también se puede proceder con la aplicación de talleres de capacitación virtual, dirigidos a padres y madres de familia, docentes y administrativos.

Fuente: Adaptado de Sibaja, Hernández y Granados. (2020). Propuesta alternativa para procesos de Práctica. Escuela de Ciencias de la Educación Universidad Estatal a Distancia.

Con base en la propuesta anterior, cada carrera debía elegir qué acciones y escenarios implementar para el desarrollo del proceso de práctica con las personas estudiantes acorde con la contextualización realizada durante el proceso de matrícula. Además, debían complementar el proceso de toma de decisiones según las distintas circunstancias, ya fueran estas resultado de las brechas digitales del estudiantado o personal docente y la disponibilidad o anuencia de las comunidades educativas y familias para participar en el proceso de práctica.

\section{SÍNTESIS Y REFLEXIONES FINALES}

En respuesta a la declaratoria de estado de emergencia decretada por el Gobierno de Costa Rica y los diferentes acuerdos tomados por el Consejo de Rectoría de la Universidad Estatal a Distancia, la Escuela de Ciencias de la Educación planteó una propuesta alternativa e innovadora, con el fin de continuar brindando oportunidades de crecimiento académico y profesional para quienes debían cumplir con el requisito de la práctica dirigida o profesional. 
Esta propuesta forma parte de la respuesta de formación que la UNED planteó ante los retos de lo que Rojas, Chan y Padilla (2019) mencionan que deben solventar los futuros profesionales para insertarse de manera exitosa en la sociedad 4.0, donde priman "el Internet de las Cosas (IoT), los cambios tecnológicos, las nuevas tendencias de consumo y la evolución de los roles que se desarrollan en la sociedad" (p. 110).

Así las cosas, este planteamiento incluyó tres posibles escenarios, la definición de los recursos y materiales básicos requeridos para su ejecución, los procesos de preparación y anticipación tanto para el estudiantado como el personal docente, además una propuesta de actividades de mediación que podían implementar las carreras según los resultados de la valoración sobre el acceso y manejo de los recursos tecnológicos por parte de las personas involucradas en la ejecución del proceso.

Cabe rescatar que la implementación de esta propuesta tomó en cuenta la brecha digital, específicamente, la relacionada con la conectividad, además de la posibilidad de que las personas estudiantes no puedan realizar la práctica en sus comunidades, por lo que se planteó la posibilidad de acudir a otras estrategias virtuales que aseguraran la vivencia de una experiencia de práctica lo más apegada posible a la realidad.

Dentro de las estrategias virtuales o escenarios planteados se incluyó el diseño de situaciones simuladas que requerirían el análisis y puesta en práctica de los aprendizajes desarrollados durante el cumplimiento del plan de estudios. Otra opción u escenario incluía complementar dichas experiencias simuladas con la aplicación en línea de exploraciones diagnósticas, desarrollo de procesos pedagógicos o talleres con distintas poblaciones según la naturaleza de cada carrera. También se dejó la opción de continuar laborando con aplicación de medidas de distanciamiento social según la estrategia del Ministerio de Educación, mediante el desarrollo de Guías para el Trabajo Autónomo según lo indicado por la DETCE (2020) y sesiones de acompañamiento virtual o presencial según las medidas sanitarias y la conectividad de la población meta lo fuesen permitiendo.

Ante el riesgo o posibilidad de que no se pudiera continuar con el apoyo de los centros de práctica o del estudiantado y sus familias, la propuesta planteó de manera preventiva la posibilidad de innovar y crear alianzas estratégicas con otros actores que se constituyeran en centros educativos aliados, también podría incluirse el reclutamiento de personas docentes que estuviesen interesadas en acoger estudiantes practicantes o incluso familias que a nivel individual manifestasen el interés en recibir apoyo del estudiantado practicante proveniente de las carreras de Educación de la UNED.

Ante el compromiso público con la continuidad de los servicios que ofrece la UNED y la necesidad de proseguir hacia la virtualización de los servicios académicos y administrativos, además del compromiso por el resguardo de la salud y el bienestar de la comunidad universitaria de la UNED, esta propuesta presentada por la Escuela de Ciencias de la Educación fue avalada en su totalidad y puesta en práctica con dos poblaciones para las que se requería realizar diferentes ajustes, por cuanto un grupo ya había iniciado y tenían procesos adelantados, mientras otro iniciaría desde cero en el segundo semestre, ya fuese porque los centros de práctica no les pudieron acoger más o porque matriculaban por primera vez esta modalidad de práctica dirigida o profesional.

Es así como esta sistematización de la propuesta permitió dejar plasmada la respuesta de la UNED ante la situación de emergencia tras la aparición del primer caso de contagio por SARS-CoV-2 y la inminente necesidad de mantener por un periodo incierto las medidas de distanciamiento social, lo que impediría continuar con las prácticas dirigidas y profesionales mediante acciones propiamente presenciales. Ello también permitirá la evaluación, mejora o réplica de esta.

Queda pendiente la evaluación de la implementación de esta propuesta según los distintos matrices que recibirá por parte de cada una de las carreras. Las experiencias del profesorado y del estudiantado al ejecutar este plan piloto serán un insumo fundamental para realimentar el proceso, redirigir acciones, asegurar nuevos recursos y plantear el camino a seguir para el I y ll semestres de 2021. 
Además, sería importante profundizar en el cómo este proceso de inmersión tecnológica ApSv ha enriquecido la experiencia de aprendizaje a distancia e incluso la forma en que podría convertirse en una opción permanente para la ejecución de prácticas dirigidas y profesionales en regiones alejadas, con diferentes grupos que se fortalezcan desde la interculturalidad y permitan el apoyo a poblaciones vulnerables, dotándoles de herramientas para el desarrollo de competencias digitales requeridas en la sociedad actual, que disminuyan las brechas existentes y les permitan mayor movilidad social.

\section{REFERENCIAS}

Adhanom, T. (2020). Alocución de apertura del Director General de la OMS en la rueda de prensa sobre la COVID-19 celebrada el 11 de marzo de 2020. Organización Mundial de la Salud (OMS). Tomado de: https://www.who.int/es/dg/speeches/detail/ who-director-general-s-opening-remarks-at-the-media-briefing-on-covid-19---11-march-2020

Asamblea Legislativa de Costa Rica. (2020). Decreto Ejecutivo N 42227 - MP - S del 16 de marzo de 2020. Recuperado de: https://www.imprentanacional.go.cr/pub/2020/03/16/ALCA46_16_03_2020.pdf

Consejo de Rectoría. (2020a). Acta de la sesión No 2078-2020, celebrada el 30 de marzo de 2020. Universidad Estatal a Distancia. [Correo para Funcionarios UNED]. Copia en posesión de Zarelly Sibaja Trejos.

Consejo de Rectoría. (2020b). Acta de la sesión No 2086-2020, celebrada el 25 de mayo del 2020. Universidad Estatal a Distancia. [Correo para Funcionarios UNED]. Copia en posesión de Zarelly Sibaja Trejos.

Consejo Universitario. (2012). Reglamento General Estudiantil. Aprobado sesión 2185, Art. V, inciso 3) de 26 de julio del 2012. Universidad Estatal a Distancia. Recuperado de: https://www.uned.ac.cr/ academica/images/cidreb/old.Reglamentos/Estudiantil/Estudiantil_Reglamento_General_24_ oct_19_f.pdf

Crespo, I. y Garrido, A. (2020). La pandemia del Coronavirus: Estrategia de Comunicación en Crisis. Más Poder Local. 41, abril 2020, pp. 12-19. ISSN: 2172-0223.

Dirección de Educacion Técnica y Capacidades Emprendedoras (DETCE) (2020). Guía de Trabajo Autónomo. Ministerio de Educación Pública. Recuperado de: http://www.detce.mep.go.cr/sites/all/ files/detce_mep_go_cr/adjuntos/guia-trabajo-autonomo-plantilla_detce_vf_15_04_2020_0.pdf

García, A., L. (2018). Blended learning y la convergencia entre la educación presencial y a distancia. RIED. Revista Iberoamericana de Educación a Distancia (2018), 21(1), pp. 09-22. DOI: http://dx.doi. org/10.5944/ried.21.1.19683 - ISSN: 1138-2783 - E-ISSN: 1390-3306

Rojas, R., Chan, H. y Padilla, K. (2019). Formación de profesionales en administración de negocios internacionales de cara a la Cuarta Revolución Industrial. Revista Nacional de Administración. Volumen 10(2), 103 - 118 Julio-diciembre.

Ruiz-Corbella, M., y García-Gutiérrez, J. (2020). Aprendizaje-Servicio en escenarios digitales de aprendizaje: propuesta innovadora en la educación superior. RIED. Revista lberoamericana de Educación a Distancia, 23, pp. 183-198. doi: http://dx.doi.org/10.5944/ried.23.1.24391

Sibaja, Z., Hernández, N. y Granados, R. (15 de mayo de 2020). Propuesta alternativa para procesos de Práctica. Escuela de Ciencias de la Educación Universidad Estatal a Distancia. [Correo para Linda Madriz]. Copia en posesión de Zarelly Sibaja Trejos.

Universidad Estatal a Distancia (2020). Sistema de Estadísticas de Matrícula:2018, 2019y 2020. Recuperado de https://estadisticasae.uned.ac.cr/estadisticaxasignatura.aspx 